\title{
INTEGRATION OF SIMULATIONS AND EXPERIMENTS FOR MODELING SUPERALLOY GRAIN GROWTH
}

\author{
Eric Payton ${ }^{1}$, Gang Wang ${ }^{1}$, Gang Wang ${ }^{1}$, Ning $\mathrm{Ma}^{1}$, Yunzhi Wang ${ }^{1}$, \\ Michael Mills ${ }^{1}$, Deborah Whitis ${ }^{2}$, David Mourer ${ }^{3}$, and Dan $\mathrm{Wei}^{3}$ \\ ${ }^{1}$ The Ohio State University; Columbus, OH 43210 USA \\ ${ }^{2}$ GE Aviation; Evendale, OH 45215 USA \\ ${ }^{3}$ GE Aviation; Lynn, MA 01905 USA
}

Keywords: grain growth, superalloys, turbine disk, deformation processing, microstructure, EBSD, phase field, René 104

\begin{abstract}
Grain size is an important parameter controlling the mechanical behavior and useful life of turbine disks. During heat treatment above the gamma prime $\left(\gamma^{\prime}\right)$ solvus the grain size increases dramatically as the $\gamma^{\prime}$ particles dissolve. The grain size then stagnates as the volume fraction of the $\gamma^{\prime}$ phase approaches zero. In general, the grain size at stagnation cannot be described by classic Zener pinning theory because of its strong dependence on the strain rate and thermal exposure during thermomechanical processing.

The grain size distribution, twin boundary fraction, $\gamma^{\prime}$ size distribution and volume fraction, carbide size distribution and volume fraction, $\gamma^{\prime}$ dissolution rates, and carbide coarsening rates have been measured in two powder metallurgical disk superalloys. Stored energy and boundary properties after deformation have been assessed. The experimental results have been used as input parameters for a physics-based model of grain growth in a field of two populations of particles, one coarsening and one dissolving. Phase field models have been used to support and clarify interpretations of experimental results and to validate the appropriate functional forms for precipitate coarsening and dissolution. Comparison of experimental and modeling results have suggested avenues for further experimental investigations and vice versa. This established feedback loop between experimentation and simulation has enabled focused experimentation, increasing the accuracy and general applicability of the model.
\end{abstract}

\section{Introduction}

Control of grain size is critically important for achieving desired mechanical properties in jet aircraft engine turbine disks. Many turbine disks are manufactured from powder metallurgy, forged below the solvus of the gamma prime $\left(\gamma^{\prime}\right)$ phase, and subsequently given a solution heat treatment above the $\gamma^{\prime}$ solvus [1]. This supersolvus heat treatment allows for in- creased control over the precipitation of the $\gamma^{\prime}$ phase during subsequent subsolvus heat treatments. The secondary and tertiary $\gamma^{\prime}$ precipitates that form during the subsolvus heat treatment are responsible for the remarkable high-temperature strength of this class of alloys [2].

Metals often retain a memory of their thermomechanical processing history through their microstructure, in the size and spatial distributions of grains, the size and spatial distributions of secondary phases, and through crystallographic texture and the distribution of boundaries of special character, such as twin boundaries. All of these factors are known to affect grain growth [3] , and the specific deformation processing conditions can affect all of these parameters [4-8]. For example, forging is usually performed at temperatures below the $\gamma^{\prime}$ solvus to take advantage of superplastic and near-superplastic behavior $[9,10]$. When the amount of grain boundary sliding relative to dislocation-based deformation accommodation is small, dislocation storage becomes significant and dynamic recrystallization may occur, altering the starting microstructure for grain growth $[11,12]$.

Computer simulations are currently used to aid in the development of optimal processing procedures; however, they are largely based on empirical data [13-15]. This prevents different alloys from being directly compared, and limits the utility of the simulations. The advancement and acceleration of alloy development requires physics-based modeling of the evolution of the microstructure during all stages of the processing operation.

\section{Experimental Procedure}

Powder metallurgical alloy René 104 [16] was selected for this investigation.

\section{Electron Backscatter Diffraction (EBSD) Analyses}

EBSD was used to measure grain size, grain size distribution, twin boundary fraction, and to assess the stored strain energy 
content of the alloy under various thermomechanical processing conditions. Double-cone specimens of as-extruded René 104 , given subsolvus heat treatments of either 1 or 30 hours at either $1052^{\circ} \mathrm{C}$ or $1121^{\circ} \mathrm{C}$ followed by compression to $70 \%$ true strain at strain rates of $0.001 / \mathrm{s}$ or $0.32 / \mathrm{s}$ at their respective heat treatment temperature, were provided by GE Aviation. The effects of strain level, strain rate, deformation temperature, and heating rate on the supersolvus heat treated grain size were evaluated. The sample dimensions for the double-cone specimen shape were the same as those used in [17]. Graphite applied in a circular trough at the ends of the samples was used to reduce barreling. Samples were then divided into quarters, with half remaining in the as-deformed condition, one quarter given an hour-long heat treatment at $8^{\circ} \mathrm{C}$ above the $\gamma^{\prime}$ solvus with a fast heating rate, and the remaining quarter given an hour-long heat treatment at the same supersolvus temperature but with a slow heating rate. A schematic of the processing and heat treatment of samples is provided in Figure 1. Samples were mounted and polished using standard metallographic techniques, and then given a vibratory polish with $0.05 \mu \mathrm{m} \mathrm{col-}$ loidal silica until the surface was suitable for EBSD. Orientation imaging microscopy (OIM), an EBSD technique, was used to measure the cross-sectional areas of at least 500 grains on each sample in accordance with ASTM standards, to characterize the grain size distributions, to characterize the $\Sigma 3$ twin boundary fractions, and to provide some crude documentation of the retained strain in the as-deformed samples through the crystallographic orientation deviation from the average orientation of each grain. The $\Sigma 3^{n}$ twin boundaries were removed in the measurement of the grain cross-sectional areas for the grain size distributions. Measurements were taken at six locations marked with microhardness indents corresponding to different strain levels, across the double-cone specimens. Four locations were along the radius of the specimen at the center of the compression axis, starting from $1 \mathrm{~mm}$ inward from the inside of the oxidation layer at the sample edge, and spaced $3 \mathrm{~mm}$ apart toward the center of the specimen. The remaining two measurements were taken $3 \mathrm{~mm}$ away from the two most internal measurements along the compression axis. All EBSD was performed on a Philips XL-30 ESEM equipped with a field emission (FEG) source. For all samples, the OIM step size was chosen such that it was less than approximately $\frac{1}{10}$ of the lineal intercept as observed from backscatter scanning electron microscopy. A 5-pixel minimum grain size was used, and a $5^{\circ}$ misorientation was chosen as the criterion for defining a grain boundary. A grain dilation cleanup was performed on the data to remove poorly indexed EBSD points.

Four samples of as-extruded material were also given subsolvus heat treatments corresponding to the same times and temperatures as above, but were not subsequently deformed. Half of each sample was given a heat treatment at $8^{\circ} \mathrm{C}$ above

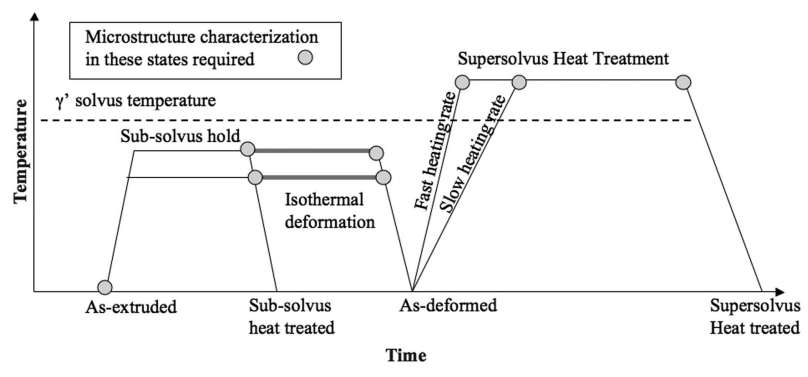

Figure 1: Temperature exposure schematic for materials investigated

the $\gamma^{\prime}$ solvus to examine grain growth in the absence of the effects of deformation. The samples from this un-deformed data set were analyzed using EBSD in the same manner as the deformed samples above.

\section{Measurements of $\gamma^{\prime}$ Particles}

The as-deformed samples were etched to reveal the $\gamma^{\prime}$ with a solution of $52 \%$ lactic acid, $26 \%$ nitric acid, and $1 \%$ hydrofluoric acid, by weight, with the remainder being water. Secondary electron imaging on a Philips Sirion FEG-SEM was used to capture digital images that were subsequently processed using Fovea Pro 3.0 and Adobe Photoshop CS in a semi-automated routine for measuring the individual cross-sectional areas, size distribution, and volume fraction.

To measure the dissolution rate of $\gamma^{\prime}$ and observe grain growth during heat treatment, interrupted supersolvus heat treatments were performed on samples approximately $1 \times 3 \times 5 \mathrm{~mm}$ at intervals from 5 seconds to 60 minutes by hanging the samples from nickel wire in a vertically-oriented tube furnace. Samples were quenched in ice water to suppress precipitation of secondary $\gamma^{\prime}$. OIM and $\gamma^{\prime}$ measurements were performed on all samples using the same methods described above.

\section{Measurements of Carbide Particles}

Carbide sizes and volume fractions were measured on polished samples using ultra-high resolution backscattered electron imaging on a Philips Sirion FEG-SEM. For each carbide size measurement, at least 50 images were obtained at magnifications greater than 5000X and subsequently processed using Fovea Pro 3.0 and Adobe Photoshop CS in a semi-automated routine. Carbide sizes measured with this technique agreed with TEM observations, and allowed for a much larger number of carbides to be measured such that a distribution of sizes could be plotted and coarsening kinetics could be experimentally measured. Carbides and borides are nearly indistinguishable using this measurement technique, however, they are expected to have the same effect on pinning the microstructure and the significant majority of particles observed are carbides $[18,19]$. The carbide coarsening kinetics were measured 
through heat treatments of as-extruded material at $1052^{\circ} \mathrm{C}$ and $1121^{\circ} \mathrm{C}$ for up to 60 hours.

\section{Additional Support Characterization}

Bright-field scanning transmission electron microscopy (BFSTEM) was performed on key samples to gauge their relative stored dislocation content and to look for signs of recrystallization using a Philips Tecnai TF-20 FEG-TEM. Some limited TEM chemical analysis, electron energy loss spectroscopy (EELS) and energy dispersive x-ray spectroscopy (EDS), was performed to look for signs of grain boundary segregation. Conventional TEM was performed to support BFSTEM observations where necessary.

OIM was performed on key TEM foils to compare with TEM observations of residual dislocation content, in the manners previously described. Backscatter SEM imaging was performed using a reverse-biased through-lens detector on the Philips Sirion FEG-SEM to compare carbide sizes and locations with those observed in BFSTEM.

Some serial sectioning was performed to support other conclusions of the study using an FEI Nova Dual-Beam instrument, equipped with both FEG and focused ion beam (FIB) sources. A $50 \times 60 \times 100 \mathrm{~mm}$ region was milled out of a thin specimen of as-extruded material that had been heat treated above the $\gamma^{\prime}$ solvus. The ion beam was used to section away successive layers of material, and backscatter imaging was performed on each layer to observe the locations of carbide particles. Thin layers of platinum were strategically deposited to reduce re-deposition of material by the ion beam near the surface being imaged.

\section{Phase Field Modeling}

The phase field method of simulating microstructure evolution is uniquely capable of capturing the evolution of important microstructural features including phase boundary character, stored energy, and dissolution and coarsening of several secondary phases simultaneously [20-23]. To describe the processes of grain growth with stable second-phase particles, the phase field method solves the time-dependent GinzburgLandau (TDGL) equation that characterizes structural changes during microstructural evolution [20]. Sets of conserved-field equations are introduced to characterize the second-phase particles. To provide insight into the differences between the growth mechanisms in 2D and 3D, a 3D phase field model capable of handling two populations of spatially heterogeneous second-phase particles was developed. The model utilized a sparse phase field algorithm, making it possible to consider large 3D systems with unique orientations for each grain [24]. Critical parameters of phase field simulation procedures will be detailed within the results section of this document as those modeling results are presented.
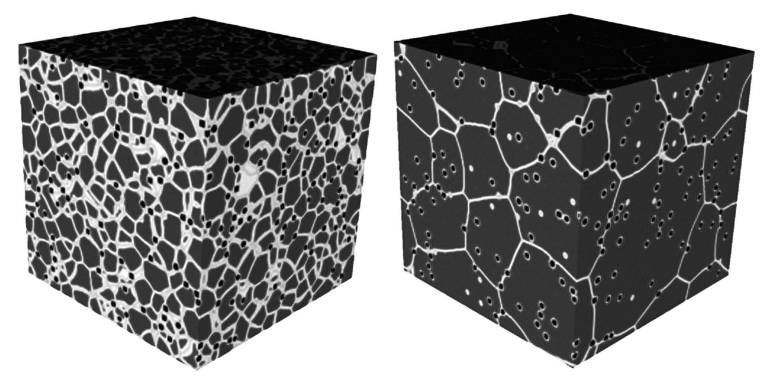

Figure 2: 3D phase field model of grain growth. Comparison of initial condition (left) with final condition (right) shows many particles become internal to grains during growth

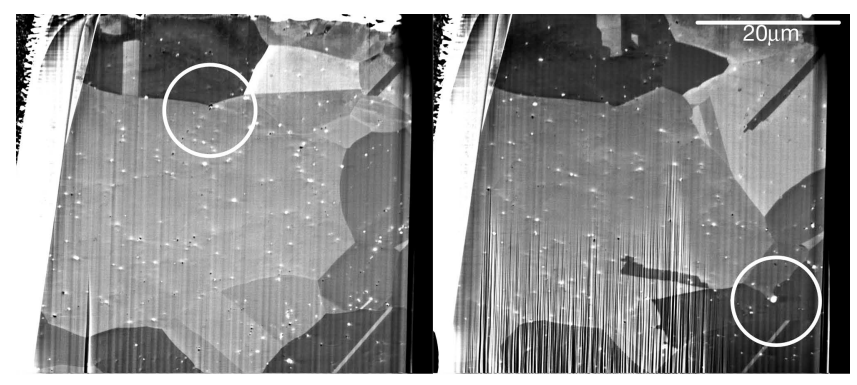

Figure 3: Backscatter SEM images from FIB serial sectioning highlighting particles pinning boundaries and showing many particles internal to grains

\section{Results}

Carbides, borides, and oxides are present at temperatures immediately above the $\gamma^{\prime}$ solvus. The existence of these particles above the $\gamma^{\prime}$ solvus limits the grain size in that temperature regime. TEM observations in this study regarding compositions, sizes, and relative fractions of the particle population above the $\gamma^{\prime}$ solvus supported the observations of Gabb et al $[18,19]$. Both SEM and TEM observations of supersolvus heat treated microstructures showed many carbides internal to the grains, as opposed to at the grain boundaries. 3D phase field modeling showed that the pinning force exerted by particles in $3 \mathrm{D}$ was sufficiently smaller than that in $2 \mathrm{D}$. Consequently, the grain boundary could break free of many more particles such that they may be found in the grain interior in planar crosssection. The initial microstructure for the phase field model was created from random noise. The system size considered was 200x200x200, with second-phase particles of radius 3 and volume fraction $5 \%$ introduced into the system randomly. Initial and final conditions from the model are shown in Figure 2. Serial sectioning results showed several clear instances of carbides or borides pinning grain boundaries, as shown in Figure 3 , but severe shadowing reduced the utility of the method for quantitative analysis.

To develop and calibrate a grain growth model, experimen- 


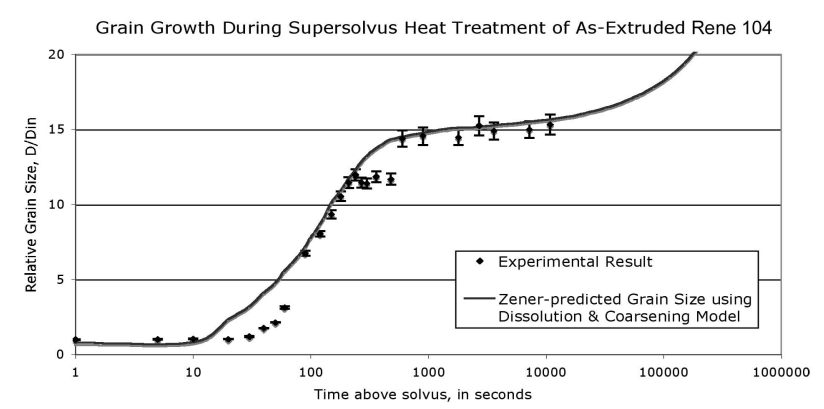

Figure 4: Comparison of experimental observations and modeling results for grain growth during supersolvus heat treatment of asextruded René 104. Grain sizes are shown relative to mean lineal intercept of as-extruded material

tal measurements of carbide coarsening, $\gamma^{\prime}$ dissolution, and grain size during heat treatment were performed. A standard cubic coarsening equation [25] was fitted to these results for introduction into the model.

Experimental results from $\gamma^{\prime}$ measurements on interrupted heat treatment samples showed that the average particle size remained approximately constant while the volume fraction changed significantly. Phase field simulations of $\gamma^{\prime}$ dissolution using a particle size distribution similar to that measured for the initial, as-extruded condition confirmed this behavior.

Interrupted heat treatment results showed that when the $\gamma^{\prime}$ particles have completely gone into solution, the grain size increases rapidly and then stagnates when boundaries impinge upon carbide particles. Using experimentally-measured carbide size and volume fraction, the grain size can be predicted fairly accurately in the absence of strain rate effects. Inclusion of carbide coarsening rates into the model supported the observation of a stagnant grain size within experimental error for the heat treatment times tested. Inclusion of $\gamma^{\prime}$ dissolution kinetics into the Zener model for particle pinning [26] reasonably agrees with the grain growth kinetics in the absence of the effects of deformation on growth as shown in Figure 4. Grain sizes in Figure 4 and elsewhere in this document are normalized with respect to the as-extruded grain size of the material.

At high temperature, grain boundary motion is much faster than $\gamma^{\prime}$ dissolution, therefore $\gamma^{\prime}$ dissolution is the rate-limiting factor in the grain growth process. The large volume fractions of $\gamma^{\prime}$ and small grain sizes at the beginning of heat treatment create a non-random correlation of particles with boundaries. Thus, even better agreement between experiments and modeling results than those shown in Figure 4 might be obtained by incorporating a correction (see, for example [27]) into the Zener pinning model. The Zener pinning model cannot, however, account for the variations in grain size observed in supersolvus heat treatments of deformed material.

Among the variables of subsolvus heat treatment time, sub- solvus heat treatment temperature, strain rate during deformation, strain level, and heating rate to $\gamma^{\prime}$ solvus, it was observed that the three factors with the greatest effect on the final mean grain size were strain rate, subsolvus heat treatment temperature, and heating rate to the solvus. The effects of strain rate were enhanced at high sub-solvus annealing and deformation temperatures. Variations in both subsolvus heat treatment temperature and heating rate to the solvus had increased effects on the grain size at low strain rates. The subsolvus heat treatment produced carbide coarsening in magnitudes that could reasonably account for the observed effects of heat treatment temperature and time on the heat treated grain size. Since deformation was performed at the same temperature as the subsolvus heat treatment, strain rate and heating rate to the solvus were responsible for producing grain size differences that could not be accounted for simply by Zener pinning from a uniform spatial distribution of carbides.

\section{Strain Rate Effects}

The strain rate of deformation is a critical parameter affecting supersolvus heat treated grain size in disk superalloys $[6,8,28]$. The effects of strain rate on grain size in René 104 are shown for two example conditions in Figure 5. Phase field simulations were constructed to analyze several observations of the key microstructural differences between samples deformed at different strain rates: stored energy in the form of dislocations, twin boundary fraction, and the coherency of $\gamma^{\prime}$. Additionally, phase field simulations of grain growth in the presence of clustered particles were performed and compared with experimental observations to determine if carbide spatial redistribution during deformation was a likely source of the observed strain rate effect.

Stored Energy from Deformation. Stored strain energy from deformation in the form of dislocation defect content is one potential source of the observed strain rate effect on the final grain size. OIM mapping on TEM foils showed that a grain reference orientation deviation (GROD) best reflected the stored dislocation content of the microstructure. Similar findings from a more sophisticated study were reported in [29]. Due to the angular resolution limitations of EBSD, and EBSD on bulk samples in particular (in which the interaction volume may encompass many dislocations and imaging conditions may vary significantly from sample to sample), quantitative measurements of stored strain are not yet possible. However, GROD analysis on OIM data seems capable of capturing a reasonable qualitative sense of the relative magnitude and spatial distribution of stored strain in the microstructure. The GROD maps and BFSTEM results for several asdeformed conditions are shown in Figure 6.

Phase field simulations were created to probe the effects of stored strain energy on grain growth. The starting microstruc- 

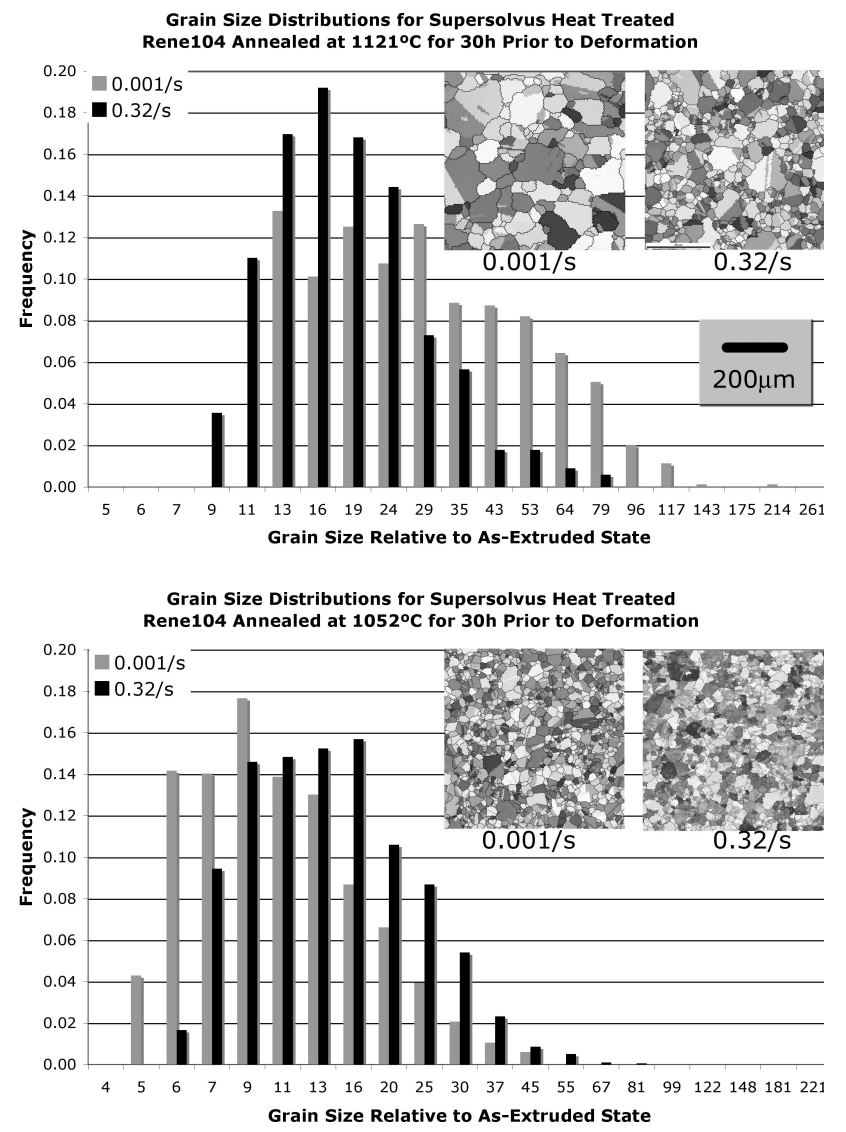

Figure 5: Strain rate during deformation is observed to have a significant effect on the supersolvus heat treated grain size. Grain size distributions are shown relative to mean lineal intercept of as-extruded material

ture for the simulations was produced from a digitized SEM image of the microstructure observed from experiments. Additional free energy was then assigned to some grains and the microstructure was allowed to evolve. The volume fraction, size, and spatial distribution of grains with extra energy were found to affect the resulting grain size. Parametric studies were performed analyzing the effects of the level of added stored energy, area fraction of grains with stored energy, and distribution of stored energy among grains of different size classes. The study of stored energy levels applied randomly to grains in the structure showed that any addition of stored energy into the system resulted in an increase in the average grain size over the uniform energy case at early stages of heat treatment, but the effect on final grain size was varied and relatively small. When only larger grains had higher stored energy, these grains experienced a smaller driving force for growth relative to the case of zero stored energy but the large grains were prevented from shrinking and disappearing by the pinning particles. The resulting effect on the final grain size was relatively small. On

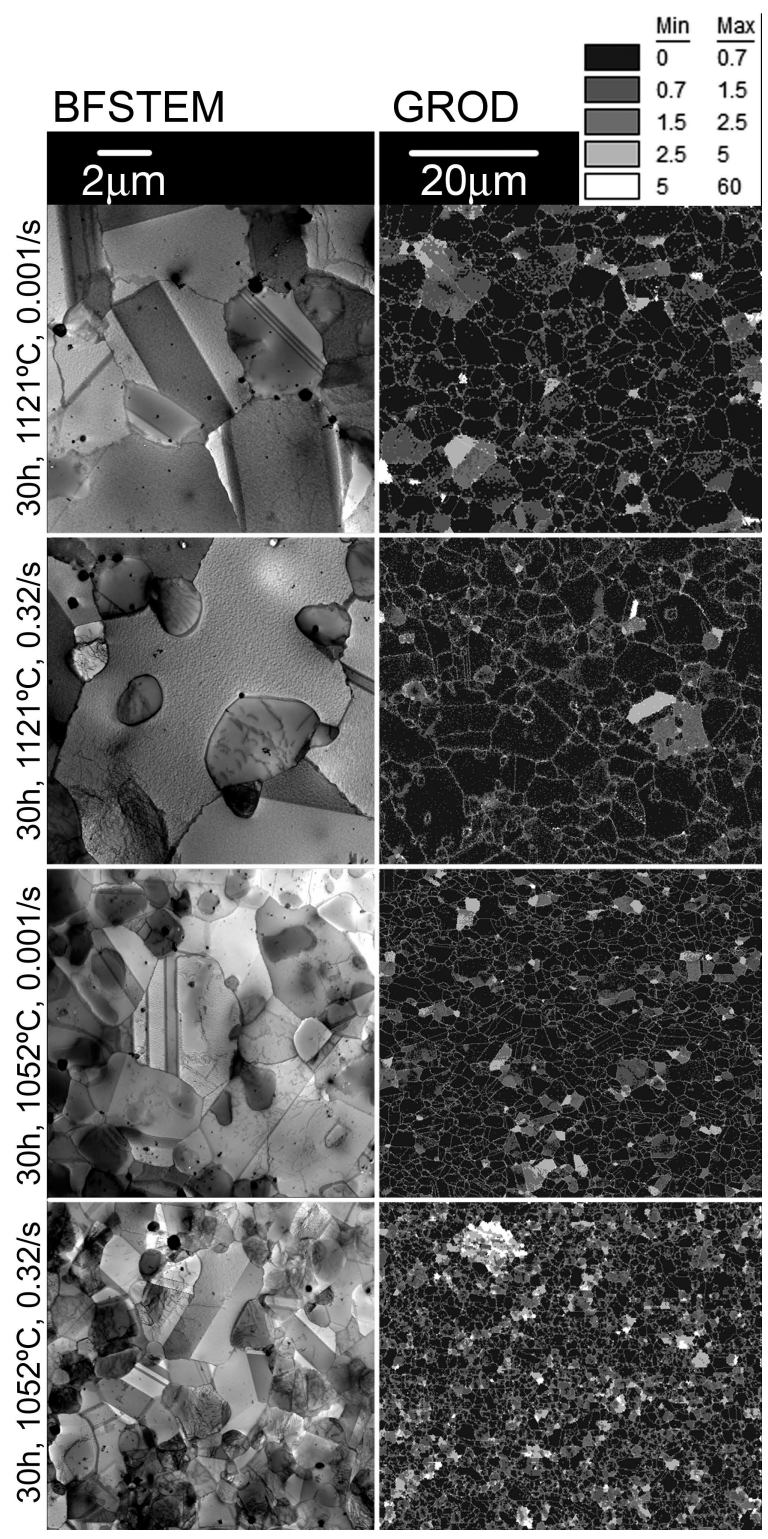

Figure 6: BFSTEM and GROD-OIM results for $30 \mathrm{~h}$ subsolvus heat treatment of as-deformed specimens. Top two rows of images correspond to the microstructures of the materials in the top plot of Figure 5 prior to heat treatment, and the bottom two rows correspond to the bottom plot of Figure 5

the other hand, when only small grains contained stored energy, the average grain diameter at stagnation increased linearly with volume fraction of stored energy. The results for the parametric study of stored energy volume fraction are shown in Figure 7. In all tested cases, the addition of spatially hereogeneous distributions of stored energy increased the final grain size.

Both EBSD and TEM observations on interrupted heat treat- 


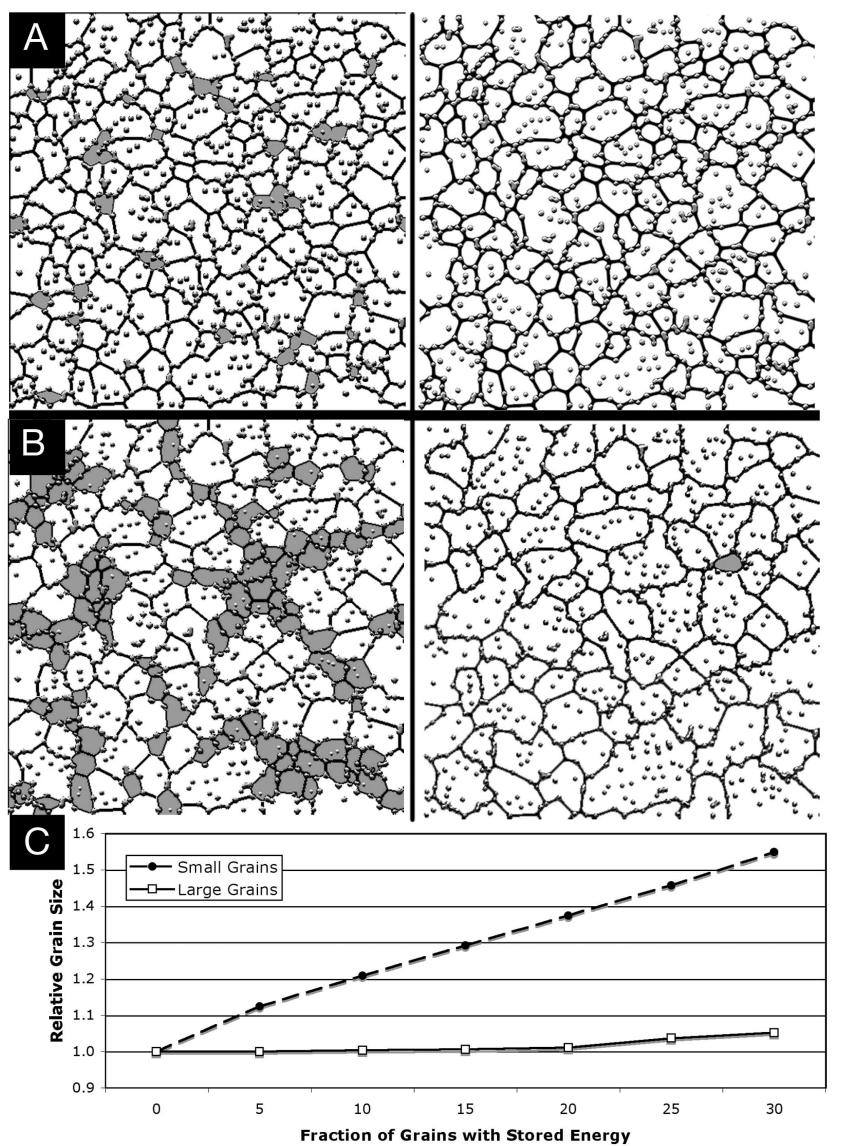

Figure 7: Phase field study of the effect of stored energy on grain growth, initial conditions on left and final structures on right: (a) 5\% volume fraction small grains given stored energy; (b) $20 \%$ volume fraction small grains given stored energy; (c) relative mean grain size vs. volume fraction of stored energy

ment samples indicated that retained strain dissipates quickly upon heat treatment, and is gone before the grain size approaches stagnation [30]. The $\gamma^{\prime}$ particles are primarily responsible for pinning the grain size below the solvus, as demonstrated by the grain growth kinetics in Figure 4. Therefore, if stored energy is responsible for any effect on the final grain size, it can only come from the effects of stored energy on the microstructure in the early stages of heat treatment.

Twin Boundary Fraction. Increases in strain rate during deformation were accompanied by a decrease in twin boundary fraction and an increase in fraction of untwinned grains both in the as-deformed and the supersolvus heat treated conditions. Figure 8 shows the average twin boundary fraction across all strain locations in the supersolvus heat treated condition. Similar observations have been previously reported in alloy 718 [31]. The presence of twin boundaries increases the

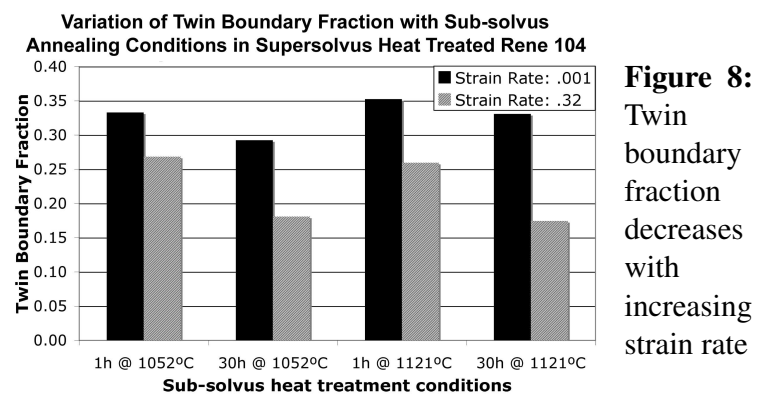

free energy of a grain. An increased fraction of twin boundaries should therefore have a limited effect on the average grain size for reasons discussed previously.

$\gamma^{\prime}$ Coherency. In René 104, the higher forging rate will generate incoherent $\gamma^{\prime}$ interfaces when the material has seen a 30 hour subsolvus heat treatment at $1121^{\circ} \mathrm{C}$, as shown in Figure 9. An incoherent $\gamma^{\prime}$ interface will increase the driving force for dissolution, accelerating the process by the Gibbs-Thompson effect [25]. The difference in dissolution rate between incoherent and coherent particles will become significant only when the particles are very small. Thus, the difference in dissolution rate between two samples with the same heating rate is probably insufficient to account for any significant difference in grain size.

The difference in boundary energy between a coherent particle and an incoherent particle should result in some differences in the pinning force exerted by the particle on the grain boundary. Figure 10 shows the phase field simulation results of a boundary interacting with a stable precipitate. If the particle is incoherent with both grains, as shown in Figure 10 (a), it will exert less pinning force than if it is coherent with the grain on the left and the boundary is being driven toward the grain on the right, as shown in Figure 10 (c). Case (b) in Figure 10 shows the opposite of (c) for comparison. The interfacial energy ratio between coherent and incoherent interfaces was assumed to be 0.1 in the phase field simulations shown in 10 .

Figure 11 compares the results of a $2 \mathrm{D}$ phase field simulation of grain growth in a field of coherent particles against grain growth in a field of incoherent particles. The initial microstructure was built from SEM experimental images. It can be seen that loss of interface coherency allows the average grain size to increase without having a significant effect on the width of the normalized grain size distribution, defined as the logarithm of grain size divided by the average of the logarithms of the grain size, $\frac{\ln A}{<\ln A>}$.

Carbide Particle Clustering. Clustering of pinning particles can also be expected to increase the average grain size [27]. During deformation at different strain rates, the carbides may be redistributed and their degree of clustering may change. Phase field simulation results also suggested that a change due 


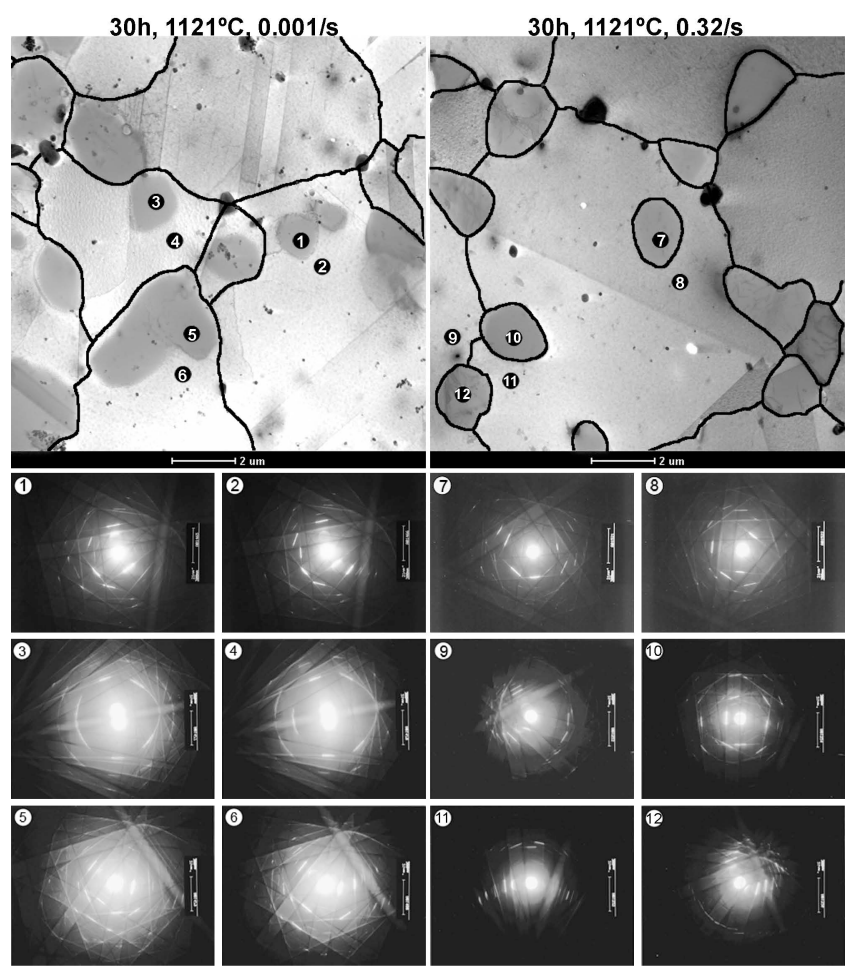

Figure 9: BFSTEM images and diffraction patterns showing coherent $\gamma^{\prime}$ particles in a sample deformed at $0.001 / \mathrm{s}$ (left) and incoherent $\gamma^{\prime}$ particles in a sample deformed at 0.32/s (right); High-angle boundaries have been outlined and $\gamma^{\prime}$ particles have been shaded for contrast

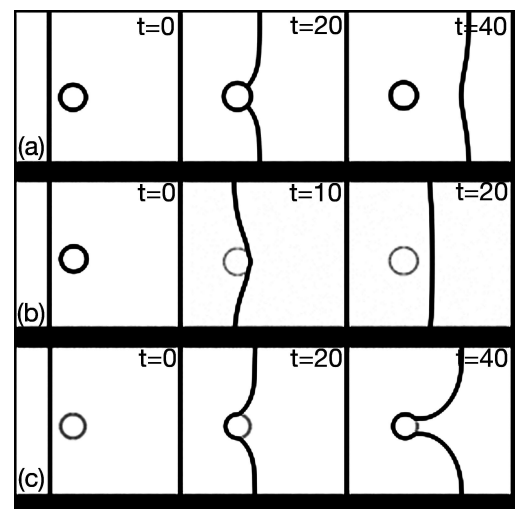

Figure 10: Interaction of a stable precipitate with a migrating boundary under three coherency states: (a) particle is incoherent with both grains; (b) particle is incoherent with the right grain but coherent with the left; (c) particle is coherent with the right grain and incoherent with the left

to clustering would result in an increase in the width of the normalized grain size distribution, as shown in Figure 12. Experimental measurements of the grain size distribution for heat treated materials in the test matrix indicated that the standard deviation of the normalized distribution was constant around 0.2 . No direct attempt was made to quantitatively characterize carbide clustering in this study.

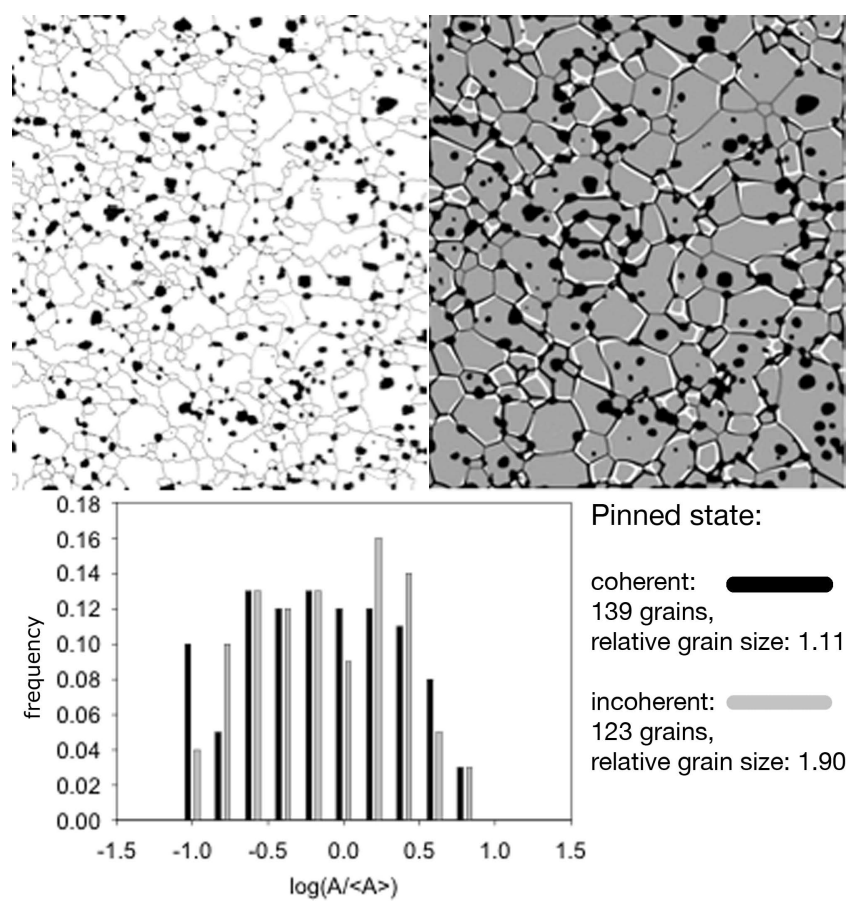

Figure 11: Phase field results of grain growth in a field of coherent precipitates vs. incoherent precipitates. Top left: Initial condition of model, imported from experimentally observed microstructure; Top right: results superimposed, where light boundaries correspond to the coherent case, dark boundaries correspond to the incoherent case, and gray boundaries are the same in both cases; Bottom: normalized grain size distributions for both coherent and incoherent cases in top right

Interfacial Properties \& Texture. Crystallographic texture can create special interfacial properties that would change the nature of grain growth [32]. All samples were analyzed for preferential grain orientations using EBSD. Crystallographic textures were not observed in any of the cases.

Additionally, it is well-known that chemical segregation at grain boundary interfaces can have a significant effect on the driving force for grain growth. EDS and EELS were performed at grain boundaries in as-extruded samples given heat treatments $8^{\circ} \mathrm{C}$ above the $\gamma^{\prime}$ solvus for $60 \mathrm{~s}, 90 \mathrm{~s}$, or 3600s. Segregation was not observed in these experiments at the grain boundaries in this alloy. Segregation of boron to grain boundaries has been observed in Ni-base superalloys through atom probe tomography on a very short length scale, below the resolution limits of the EDS and EELS performed in this study [33]. It remains possible that differences at the grain boundaries caused by the deformation accommodation mechanisms, namely the degree of grain boundary sliding experienced by the alloy, could give rise to a change in the amount of solute segregation at the boundaries and result in a difference in mobility or grain boundary energy that affects grain growth. How- 

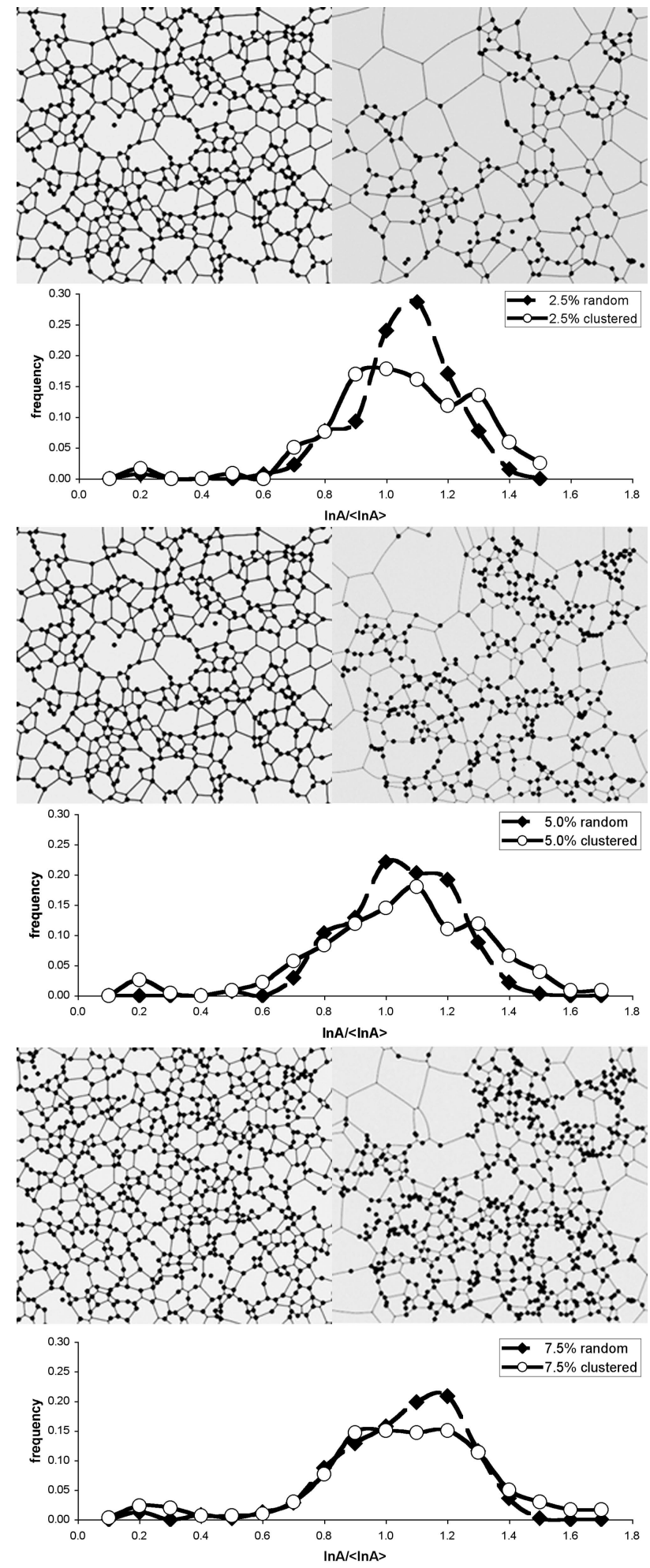

Figure 12: Phase field model results showing normalized grain size distributions for different volume fractions of pinning particles, distributed randomly on left and clustered on right: Top: $2.5 \%$, Middle: $5.0 \%$, Bottom: $7.5 \%$ ever, bulk diffusion is more important at temperatures as high as the $\gamma^{\prime}$ solvus, so the segregation becomes less likely as a source of the strain rate effect.

Heating Rate to the $\gamma^{\prime}$ Solvus

As discussed previously, coherency of $\gamma^{\prime}$ should have a significant effect on the rate of dissolution of the $\gamma^{\prime}$ particles only when they are small and close to completely dissolving. A widespread change in $\gamma^{\prime}$ coherency was observed only for samples deformed at the higher strain rate, but the effect of heating rate to the $\gamma^{\prime}$ solvus was strongest at low strain rates. It is therefore unlikely that differences in $\gamma^{\prime}$ dissolution due to coherency are the source of the heating rate effect. It has been suggested that fast dissolution leads to more 'normal' grain growth, while slow dissolution leads to more abnormal or discontinuous grain growth [34].

\section{Discussion}

When implemented into phase field simulations, microstructural differences between samples deformed at different strain rates were not individually able to account for the observed differences in heat treated grain sizes. The strain rate effect therefore must be accounted for by another aspect of the grain growth behavior in this alloy. Normal grain growth is a continuous annealing phenomenon in which the general appearance of the microstructure at different snapshots in time appear similar at scaled magnifications. In normal grain growth, this self-similarity may be quantified by a constant value of the standard deviation of the natural logarithm of the grain size measurement (either cross-sectional area or lineal intercept) normalized by the average value of the natural logarithm of the measurement, $\frac{\ln A}{<\ln A>}$ [35]. If the standard deviation of the normalized grain size distribution varies during growth, then the growth is not self-similar, and is thus a discontinuous annealing phenomenon. Grain growth in the presence of precipitates produces a discontinuous annealing phenomenon [3]. Experimental measurements on as-deformed material compared with the corresponding supersolvus heat treated material showed large variations in this self-similarity parameter. Phase field results and interrupted heat treatment experiments on as-extruded material showed that the standard deviation of the normalized grain size distribution varied during $\gamma^{\prime}$ dissolution but was necessarily constant when the grain size stagnates. Figure 13 shows the results of this analysis as applied to a heat treatment of as-extruded material and the consequence of the observed variation in self-similarity parameter on the cumulative grain size distribution.

Discontinuous grain growth phenomena have previously been identified in other Ni-base disk superalloy systems [17, 36-38]. Song directly observed the broadening of the grain size distribution during heat treament in IN100 via a change in 


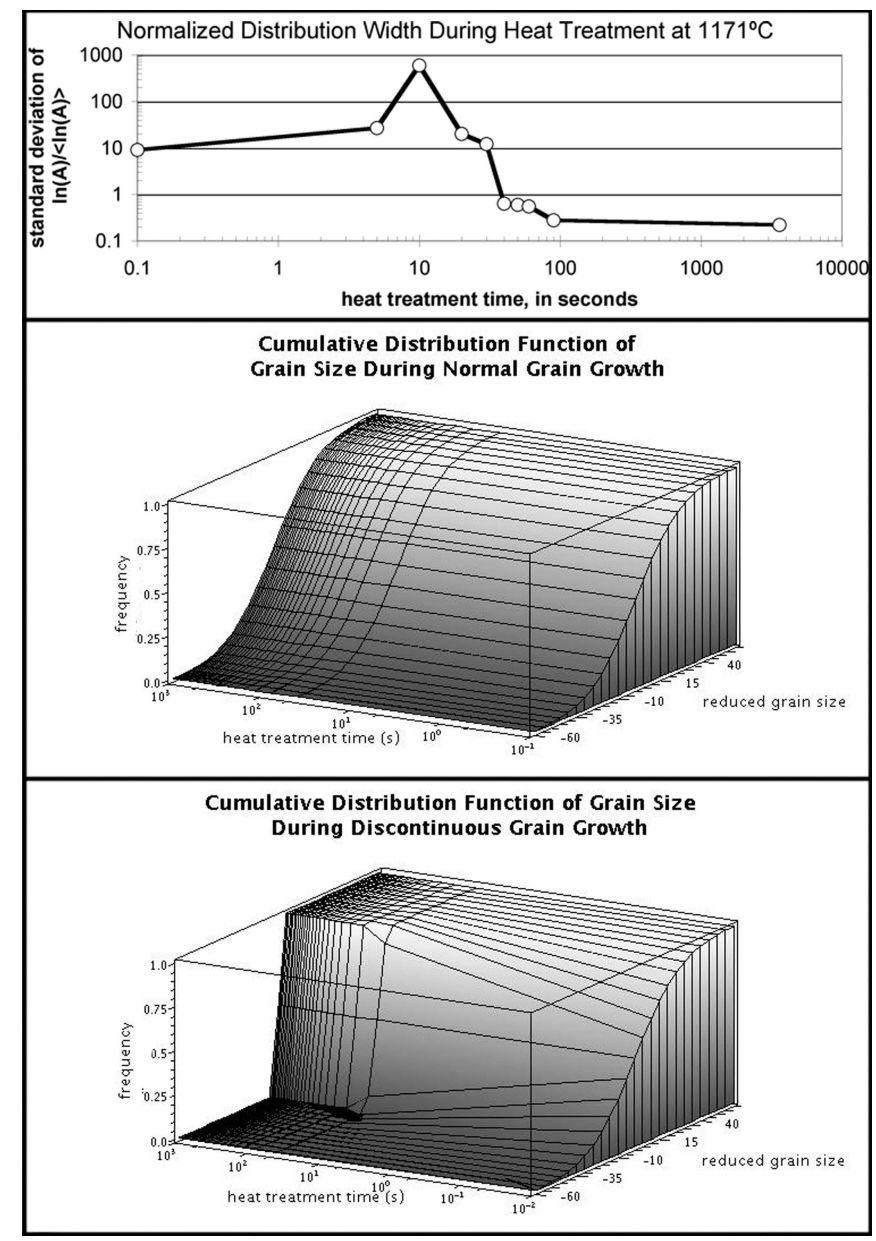

Figure 13: Normalized distribution width changes during heat treatment, indicating discontinuous grain growth (top); Normal grain growth is characterized by a constant reduced grain size distribution (middle), but the reduced grain size distribution does not have a constant shape in discontinuous grain growth (bottom)

mean grain boundary curvature [37] and the ratio of the maximum grain size to the average grain size [38]. The broadening of the grain size distribution indicates some degree of abnormal grain growth is occurring. Interrupted heat treatments of samples that have been deformed at strain rates giving significantly different final heat treated grain sizes may be able to provide greater insight into the source of the observed strain rate effect.

\section{Conclusions}

The results of this investigation have indicated that crystallographic texture, changes in twin boundary fraction, differences in $\gamma^{\prime}$ coherency, pinning particle spatial redistribution, and differences in the driving force for grain growth resulting from differences in stored energy from deformation are, indi- vidually, not likely to be responsible for the observed effect of strain rate on the supersolvus heat treated grain size in these alloys. Analysis of experimental results have indicated that the evolution of the grain size is not uniform in microstructure during thermomechanical processing.

The field of dissolving $\gamma^{\prime}$ particles prevents normal, selfsimilar grain growth from occurring during supersolvus heat treatment in Ni-base disk superalloys. Predictions of the stagnation grain size according to classical Zener pinning theory are unsatisfactory because they cannot account for the observed strain rate effect. Stored energy may play a small role in determining the final grain size, but cannot be solely responsible for the strain rate effect, as the stored energy dissipates faster than the growth occurs. Simulations suggest that the observed changes in twin boundary fractions should reduce the observed effect in material deformed at high temperatures. Clustering of pinning particles does not appear to occur because the normalized width of the grain size distribution is essentially constant after heat treatment for various deformation conditions. Dissolution rates of $\gamma^{\prime}$ may be responsible for the effects of heating rate by changing the force balance between Zener drag and the driving force for grain boundary motion during discontinuous grain growth.

\section{Acknowledgements}

This work was sponsored by GE Aviation and performed at The Ohio State University. The authors wish to thank both entities for their respective support while carrying out this work.

\section{References}

[1] D. Furrer and H. Fecht, "Ni-based Superalloys for Turbine Discs," JOM, 51(1) (1999), 14-17.

[2] M. J. Donachie and S. J. Donachie, Superalloys: A Technical Guide, 2nd ed. (Materials Park, OH: ASM International, 2002).

[3] F. J. Humphreys, "A Unified Theory of Recovery, Recrystallization, and Grain Growth, Based on the Stability and Growth of Cellular Microstructures - II. The Effect of Second-Phase Particles," Acta Materialia, 45(12) (1997), 5031-5039.

[4] M. O. Alniak and F. Bedir, "Change in Grain Size and Flow Strength in P/M René 95 Under Isothermal Forging Conditions," Materials Science and Engineering B, 130 (2006), 254263.

[5] J. W. Brooks, "Forging of Superalloys," Materials and Design, 21 (2000), 297-303.

[6] E. Huron, S. Srivatsa, and E. Raymond, "Control of Grain Size Via Forging Strain Rate Limits for René 88 DT," Superalloys 2000, ed. T. M. Pollock et al. (Warrendale, PA: TMS, 2000), 49-58.

[7] A. R. Mashreghi et al., "High Temperature Deformation of Nickel Base Superalloy Udimet 520," Materials Science and Technology, 20(2) (2004), 161-168. 
[8] T. Matsui et al., "The Microstructure Prediction of Alloy $720 \mathrm{Li}$ for Turbine Disk Applications," Superalloys 2000, ed. T. M. Pollock et al. (Warrendale, PA: TMS, 2000), 127-134.

[9] C. P. Blankenship et al., "Hot-Die Forging of P/M Ni-Base Superalloys," Superalloys 1996, ed. R. D. Kissinger et al. (Warrendale, PA: TMS, 1996), 653-663.

[10] M. Durand-Charre, The Microstructure of Superalloys (Amsterdam: Overseas Publishers Association, 1997).

[11] J.-P. A. Immarigeon and P. H. Floyd, "Microstructural Instabilities During Superplastic Forging of a Nickel-base Superalloy Compact," Metallurgical Transactions A, 12A(7) (1981), $1177-1186$.

[12] O. Kaibyshev, Superplasticity of Alloys, Intermetallics, and Ceramics (New York: Springer-Verlag, 1992).

[13] B. Antolovich and M. Evans, "Predicting Grain Size Evolution of UDIMET alloy 718 During the "Cogging" Process Through the Use of Numerical Analysis," Superalloys 2000, ed. T. M. Pollock et al. (Warrendale, PA: TMS, 2000), 437-446.

[14] C. U. Hardwicke and G. Shen, "Modeling Grain Size evolution of P/M René 88 DT Forgings," Advanced Technologies for Superalloy Affordability, ed. K.-M. Shang et al. (Warrendale, PA: TMS, 2000), 265-275.

[15] M. Stockinger and J. Tockner, "Optimizing the Forging of Critical Aircraft Parts by the Use of Finite Element Coupled Microstructure Modeling," Superalloys 718, 625, 706 and Various Derivatives, ed. E. A. Loria (Warrendale, PA: TMS, 2005), 8794.

[16] D. P. Mourer et al., "Ni Based Superalloy and its Use as Gas Turbine Disks, Shafts, and Impellers," (European Patent EP 1,195,446 A1: General Electric Company, 2002).

[17] D. D. Whitis, "Recovery and Recrystallization After Critical Strain in the Ni-base Superalloy René 88 DT," Superalloys 2004, ed. K. A. Green et al. (Warrendale, PA: TMS, 2004), 391400.

[18] T. P. Gabb et al., "Detailed Microstructural Characterization of the Disk Alloy ME3," (TM-2004-213066: NASA Glenn Research Center, 2004).

[19] T. P. Gabb, A. Garg, and D. L. Ellis, "Microstructural Evaluations of Baseline HSR/EPM Disk Alloys," (NASA TM-2004213123: NASA Glenn Research Center, 2004).

[20] L.-Q. Chen, "Phase-field Models for Microstructure Evolution," Annual Review of Materials Research, 32 (2002), 113-140.

[21] D. N. Fan and L.-Q. Chen, "Diffuse-interface Description of Grain Boundary Motion,” Philosophical Magazine Letters, 75 (1997), 187-196.

[22] K. Thornton, J. Agren, and P. W. Vorhees, "Modelling the Evolution of Phase Boundaries in Solids at the Meso- and NanoScales," Acta Materialia, 51 (2003), 5675-5710.

[23] J. Wang et al., "Phase-field modeling with CALPHAD and CVM for Microstructural Evolution of Ni-base Superalloy," Superalloys 2004, ed. K. A. Green et al. (Warrendale, PA: TMS, 2004), 933-940.
[24] J. Gruber et al., "Sparse Data Structure and Algorithm for the Phase Field Method," Modeling and Simulation in Materials Science, 14(7) (2006), 1189-1196.

[25] D. A. Porter and K. E. Easterling, Phase Transformations in Metals and Alloys, 2nd ed. (Cheltenham, UK: Nelson Thornes, LTD, 1992).

[26] P. A. Manohar, M. Ferry, and T. Chandra, "Five Decades of the Zener Equation,” ISIJ International, 38(9) (1998), 913-924.

[27] F. J. Humphreys and M. Hatherly, Recrystallization and Related Annealing Phenomena, 2nd ed. (Tarrytown, NY: Elsevier Science, Inc, 1996).

[28] H. S. Jeong, J. R. Cho, and H. C. Park, "Microstructure Prediction of Nimonic 80A for Large Exhaust Valve During Hot Closed Die Forging," Journal of Materials Processing Technology, 162-163 (2005), 504-511.

[29] M. Kamaya, A. J. Wilkinson, and J. M. Titchmarsh, "Quantification of plastic strain of stainless steel and nickel alloy by electron backscatter diffraction," Acta Materialia, 54 (2006), 539-548.

[30] D. R. Corwin, "Characterization of Microstructure Evolution of Nickel Base Superalloys in Support of the Development of a Grain Growth Model,' (Master's thesis: The Ohio State University, 2007).

[31] J.-P. Thomas et al., "EBSD Investigation and Modeling of the Microstructural Evolutions of Superalloy 718 During Hot Deformation," Superalloys 2004, ed. K. A. Green et al. (Warrendale, PA: TMS, 2004), 959-968.

[32] L. S. Shvindlerman and G. Gottstein, "Cornerstones of Grain Structure Evolution and Stability: Vacancies, Boundaries, Triple Junctions," Journal of Materials Science, 40 (2005), 819-839.

[33] M. K. Miller, "Contributions of Atom Probe Tomography to the Understanding of Nickel Base Superalloys," Micron, 32(8) (2001), 757-764.

[34] P. R. Rios, "Abnormal Grain Growth from Uniform Grain Size Distributions," Acta Materialia, 45(4) (1997), 1785-1789.

[35] J. W. Martin, R. D. Doherty, and B. Cantor, Stability of Microstructure in Metallic Systems, 2nd ed.Cambridge Solid State Science Series (New York, NY: Cambridge University Press, 1997).

[36] Y. K. Cho, D. Y. Yoon, and M. F. Henry, "The Effects of Deformation and Pre-Heat-Treatment on Abnormal Grain Growth in René 88 Superalloy," Metallurgical and Materials Transactions A, 32A(12) (2001), 3077-3090.

[37] K. Song, "Grain Growth Phenomena in P/M Nickel-base Superalloys," (Ph.D. thesis: University of Connecticut, 2005).

[38] K. Song and M. Aindow, "Stability of Second-Phase Particles and Abnormal Grain Growth in Superalloy IN100," Materials Science and Technology 2006: Phase Stability, Diffusion, and their Applications, vol. 2, ed. Z.-K. Liu et al. (Warrendale, PA: TMS, 2006), 211-220. 